\title{
Development of a Scottish physical activity questionnaire: a tool for use in physical activity interventions
}

\author{
Matthew Lowther, Nanette Mutrie, Christopher Loughlan, Catriona McFarlane
}

\begin{abstract}
Objectives-Three studies were undertaken to establish the reliability and validity of the Scottish physical activity questionnaire (SPAQ), developed to aid seven day recall of leisure and occupational physical activity.
\end{abstract}

Methods-To establish reliability, SPAQs $(n=34)$ were completed on a Monday and the following Wednesday. Thus each questionnaire measured four identical days. To establish concurrent validity, 94 participants completed a SPAQ and an adapted stage of exercise behaviour change questionnaire. Responses to SPAQ were then analysed by stage of exercise behaviour change. In a further study of criterion validity, 30 volunteers wore a Caltrac motion sensor for four consecutive days, after which they completed a SPAQ.

Results-In the first study, total physical activity had a coefficient of repeatability $(R)$ of 53 minutes. Occupational physical activity showed a similar variance $(R=$ 54.6 minutes) but leisure physical activity was more reliable $(R=29.3$ minutes $)$. The main variation in occupational physical activity was found to be walking $(R=39.8$ minutes). In study 2 , a one way analysis of variance showed the expected relation between physical activity and stage of exercise behaviour change, confirming the concurrent validity of SPAQ with the stage of exercise behaviour change model. In study 3, several erroneous recordings affected both SPAQ and the Caltrac results (kcal). After relevant corrections had been made, the correlation between the two measurement devices was $0.52(\mathrm{p}<0.05)$. Conclusions-SPAQ has been shown to be reliable and to hold strong concurrent validity and limited criterion validity. The main limitation in SPAQ appears to be the measurement of occupational walking. It is therefore recommended that further work be conducted to refine the measurement of this physical activity component. It is evident nonetheless that SPAQ can be used with confidence to measure outcomes in physical activity interventions when account is taken of its limitations. (Br F Sports Med 1999;33:244-249)

Keywords: physical activity; questionnaire; reliability; validity

There is growing evidence to suggest that participation in physical activity (described as "any bodily movement produced by skeletal muscles that results in energy expenditure"1) may have several benefits for health and protect against certain chronic diseases such as coronary heart disease, ${ }^{2}$ hypertension, ${ }^{3}$ non-insulindependent diabetes mellitus, ${ }^{4}$ certain site specific cancers, ${ }^{5}$ and osteoporosis, ${ }^{6}$ as well as normalising fat metabolism ${ }^{7}$ and increasing energy expenditure aiding weight control. ${ }^{8}$ National surveys carried out in England, ${ }^{9}$ Northern Ireland, ${ }^{10}$ and Scotland ${ }^{11}$ indicate very high levels of inactivity, with 34,38 , and $53 \%$ of the English, Northern Irish, and Scottish populations respectively reporting only one to four occasions of a mix of moderate and vigorous activity or less in the preceding month. It can be concluded that a high proportion of the British population can be targeted with interventions aimed at increasing physical activity.

The Scottish physical activity questionnaire (SPAQ) was developed to measure two key constructs: stage of exercise behaviour change and seven day recall of physical activity.

\section{Methods and results}

MEASURING BEHAVIOUR CHANGE

The "stages of behaviour change" model was introduced by Proshaska and DiClemente ${ }^{12}$ for describing the stages people go through when attempting to change negative behaviours such as smoking. This model was adapted for use in exercise studies by Marcus et $a l^{13}$ to describe five stages that a person moves through from being sedentary to regularly active. These stages are precontemplation (regularly inactive and no intention of change), contemplation (regularly inactive, but intending to change in next six months), preparation (active, but not regularly), action (regularly active but only in last six months) and maintenance (regularly active for longer than six months). A recent review has reported over 25 studies using this model to research exercise behaviour. ${ }^{14}$

Marcus et $a l^{13}$ originally suggested that "regular" exercise meant three or more sessions each week for at least 20 minutes per session. This definition does not take account of the most recent suggestions from the American College of Sports Medicine ${ }^{15}$ or UK recommendations ${ }^{16}$ that sedentary people should aim to accumulate 30 minutes of activity on most days of the week. Loughlan and Mutrie ${ }^{17}$ have adapted the definition to include general physical activity such as walking, gardening, etc, and Mutrie et $a l^{18}$ have reported $28-40 \%$ in maintenance and $35-55 \%$ in contemplation and preparation stages from various populations such as NHS staff, students, and diabetic patients. 
MEASURING PHYSICAL ACTIVITY

There are numerous methods (objective and subjective) of measuring physical activity, each with characteristic advantages and disadvantages. Objective measures such as heart rate monitoring, radioisotope analysis, and motion sensor analysis can provide very detailed accurate measurement of physiological variables (which can be converted into relevant units such as energy expenditure) and have been shown to be highly reliable and valid measures of physical activity. ${ }^{19}{ }^{20}$ However, the equipment involved in these studies can often be expensive, making the cost of large scale studies prohibitive. ${ }^{21}$ In addition, the use of the more objective measures of physical activity often require considerable researcher interaction, which can create a source of bias and they often need to be conducted in the laboratory for accurate measurement ${ }^{22}$ making their use in large field studies unrealistic.

The use of diary and recall methods are commonplace subjective forms of measuring physical activity, as they are relatively cheap to produce and administer and can be used to measure physical activity in field studies. However, a recent report highlighted the need for researchers to tackle the area of validating and standardising the existing tools. ${ }^{23}$ With respect to physical activity recall questionnaires, Laporte et $a l^{1}$ concluded that they have been found to be accurate, non-reactive, and practical for use with large subject numbers. One such questionnaire is the Stanford seven day activity recall questionnaire, ${ }^{24}$ which was developed to record the duration and intensity of recent occupational and leisure time physical activity. Although its validity and reliability have been established, ${ }^{24}$ the language is North American culture bound and it needs to be administered by interview. Consequently, it was decided to amend this questionnaire so it could be used in postal surveys with a British population.

\section{DEVELOPMENT OF SPAQ}

As SPAQ was originally developed to measure changes in the leisure physical activity of various groups, the leisure component of the Stanford questionnaire was used to devise the first versions of SPAQ. It was also a basic requirement of these initial studies that in some cases the questionnaire was to be administered by post. As Blair $e t a l^{4}$ state that the need for the Stanford physical activity questionnaire to be interview led was to "help participants estimate the intensity of various activities", it was considered that simplifying the intensity component of the questionnaire would allow it to be used in postal surveys. The Stanford physical activity questionnaire requires participants to estimate the number of hours in the preceding week spent in either sleep or moderate, hard, or very hard physical activity. It was decided to simplify this for SPAQ by asking participants only to include activities that are at least of moderate intensity or above (they are given a list of activities that may or may not be included). After further minor textual changes, initial versions of SPAQ were extensively piloted, revised, and applied in the research field. ${ }^{25}$
In order for SPAQ to be able to take account of the current physical activity reccomendations, ${ }^{15}{ }^{16}$ it was restructured to include a leisure time and occupational physical activity section, with each section containing questions on general activity such as walking, stair climbing, manual labour, etc. In this way, total physical activity could be calculated. Although SPAQ does not attempt to measure energy expenditure directly, it does record all activities of at least moderate intensity or above. In this respect, it would be fair to assume that total physical activity measured by SPAQ is related to total energy expenditure. A final addition to SPAQ was a section for the measurement of the stages of exercise behaviour change, which consisted of the adapted stages of exercise behaviour change questionnaire of Loughlan and Mutrie. ${ }^{17}$ In the next sections are described three studies undertaken to establish the reliability (study 1 ) and validity (studies 2 and 3 ) of the final version of SPAQ.

RELIABILITY OF SPAQ (STUDY 1)

It was the aim of this study to evaluate the testretest reliability of SPAQ.

Thirty four people participated. Eighteen (mean (SD) age 33 (12) years; two men, 16 women) were regular members of an aerobics class, and 16 (mean (SD) age 33 (11.1) years; seven men, nine women) were members of the public who had volunteered for an exercise promotion project.

Fifty four SPAQs were distributed to the members of the aerobics class and 46 to the general public group. Participants were asked to complete it on a Monday (test) and again on the following Wednesday (retest). As SPAQ is a seven day recall questionnaire, this resulted in both test and retest questionnaires measuring the same four days of physical activity. Two distinct groups were targeted in an effort to assess the reliability of SPAQ across a broad range of physical activity.

The relation between test and retest questionnaires was initially assessed using a Pearson correlation coefficient. However, Bland and Altman ${ }^{26}$ have queried the use of the correlation coefficient for use in reliability studies and have suggested the use of the coefficient of repeatability. This coefficient was also calculated in the present study to assess test-retest reliability.

A total of 53 questionnaires were initially returned giving a response rate of $53 \%$. Of these, 35 of the retest questionnaires were returned giving an overall response rate of $35 \%$. One retest questionnaire differed from the test questionnaire by over five hours; this difference was more than 10 times the standard deviation of the mean differences of the remaining 34 data sets (26.5 minutes) and was therefore excluded from the analysis. Table 1 gives descriptive statistics for all participants minus this exclusion.

Figure 1 shows the relation between test and retest results. The relation appears to be very strong, with a correlation coefficient of 0.998 , showing it to be significant (critical value $0.436, \mathrm{p}<0.01 ; 32 \mathrm{df}$ ). However, Bland and Altman ${ }^{26}$ have queried the use of the product- 
Table 1 Descriptive statistics for test and retest questionnaire responses measured over the four overlapping days displayed in minutes

\begin{tabular}{lllllll}
\hline \multirow{2}{*}{$\begin{array}{l}\text { Measurement } \\
\text { questionnaire }\end{array}$} & \multicolumn{7}{l}{ Descriptive statistics } & (minutes) & & \\
\cline { 2 - 7 } & $N$ & Mean & Median & $S D$ & Min & Max \\
\hline Test & 34 & 587.5 & 437.5 & 464.6 & 45.0 & 1950.0 \\
Retest & 34 & 590.6 & 425 & 469.1 & 55.0 & 1960.0 \\
\hline
\end{tabular}

moment correlation coefficient $(r)$ for determining reliability and have suggested the use of the coefficient of repeatability $(R)$.

The first stage in calculating $R$ is to plot the differences between the test and retest results against the average of the test and retest results for each subject (fig 2).

The next step is to calculate the mean and standard deviation of the differences. For the present study, the mean difference is 3.09 minutes and the standard deviation 26.5 minutes. As the same method of measurement was used, the mean difference should not be significantly different from zero (if it was, the data could not be used for analysis as clearly they would not be reliable). ${ }^{26} \mathrm{~A}$ one sample $t$ test confirmed no significant difference. Under a normal distribution, $95 \%$ of the differences should be less than 2 standard deviations, this being adopted by the British Standards Institution ${ }^{27}$ as the definition of $R$. As can be seen from fig 3, the differences follow a normal distribution (an Anderson-Darling normality test confirmed this; $\mathrm{A}^{2}=0.436, \mathrm{p}=0.281$ ) and, as we have already established, are not significantly different from zero; $R$ for this study is 53 minutesthat is, twice the standard deviation of the differences. This means that $95 \%$ of the differences in SPAQ from one measurement to the next (under similar conditions) would be between zero plus or minus 53 minutes.

We examined $R$ for the occupational and leisure time sections of SPAQ separately to assess

Figure 1 Relation between test-retest questionnaires for all participants displayed in minutes.

Figure 2 Difference in test-retest results (minutes) plotted against the mean scores for the test and retest results (minutes).
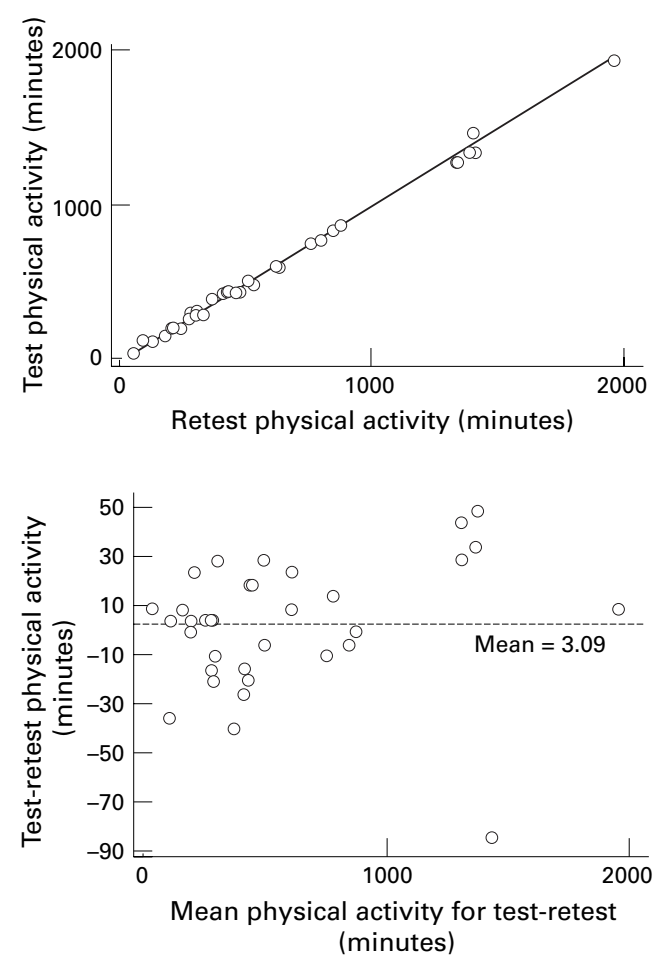

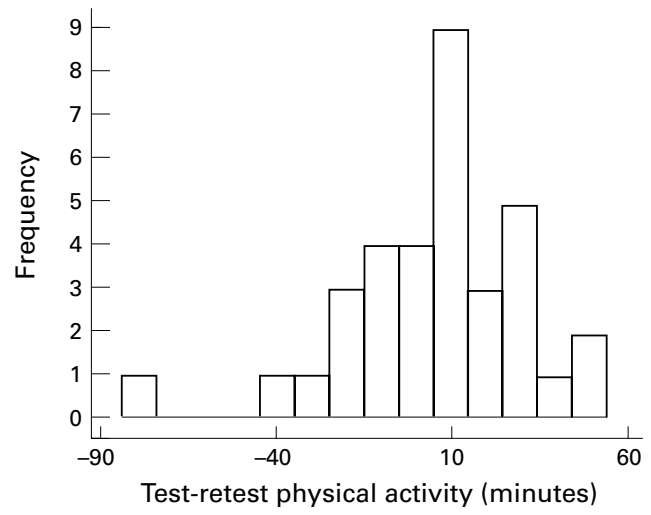

Figure 3 Distribution of differences for test-retest questionnaires (minutes).

if either was responsible for the potentially large variation in total physical activity. We found that $R$ for leisure physical activity was 29.3 minutes compared with 54.6 for occupational physical activity, suggesting the latter was largely responsible for the variation in total physical activity. Analysis of each separate component of occupational physical activity showed that housework had an $R$ of 20.2 minutes, manual labour 13.6 minutes, and walking 39.8 minutes. It is clear therefore that the variation in occupational walking was largely responsible for the large variation in total physical activity. In addition, if only leisure time physical activity is used, SPAQ shows less than 30 minutes of variation.

\section{VALIDITY OF SPAQ}

Study 2

It was the aim of this study to show the concurrent validity of SPAQ using the adapted version of the stage of exercise behaviour change questionnaire. ${ }^{17}$

In order to collect data for all the stages of exercise behaviour change, a range of subject groups were targeted. A total of 94 people participated in the study which included 29 regular members of an aerobics class (mean (SD) age 33 (10.8) years), 44 members of the public who had volunteered for an exercise project (mean (SD) age 35 (12.2) years), and 21 users of a local community centre (mean (SD) age 35 (11.8) years).

Fifty four SPAQs were distributed to members of the aerobics class, 96 to the general public group, and 50 to the community centre users. Participants were asked to complete both the stage of exercise behaviour change section and the physical activity seven day recall section.

A one way analysis of variance was used to identify significant differences between each stage of exercise behaviour change for total physical activity, leisure time physical activity, and occupational physical activity. This analysis was repeated with the appropriate nonparametric test, the Kruskal-Wallis test. The Fisher multiple comparison test was used for follow up analysis.

A total of $94(47 \%)$ questionnaires were returned, 29 (54\%) from the aerobics class, 44 (46\%) from the general public, and $21(42 \%)$ from the community centre group. Table 2 
Table 2 Descriptive statistics for total physical activity (minutes/week) for each stage of change

\begin{tabular}{lrcccccc}
\hline \multirow{2}{*}{ Stage of change } & \multicolumn{7}{c}{ Descriptive statistics for total physical activity } \\
\cline { 2 - 8 } & \multicolumn{1}{c}{$N$} & $\%$ & Mean & Median & SD & Min & Max \\
\hline Precontemplation & 9 & 10 & 452 & 450 & 248 & 105 & 750 \\
Contemplation & 15 & 16 & 395 & 320 & 243 & 70 & 800 \\
Preparation & 13 & 14 & 672 & 645 & 307 & 245 & 1260 \\
Action & 21 & 22 & 1016 & 870 & 547 & 360 & 2540 \\
Maintenance & 36 & 38 & 1234 & 917 & 835 & 310 & 3390 \\
\hline
\end{tabular}

gives descriptive statistics for the total amount of physical activity (minutes a week) for each stage of exercise behaviour change. It shows that the ratio of respondents in each of the stages of exercise behaviour change was similar to comparable research, ${ }^{18}$ suggesting a representative sample. A one way analysis of variance confirmed that significant interstage differences existed for reported weekly physical activity $(F[4,89]=7.19, \mathrm{p}<0.05)$. A KruskalWallis non-parametric test was also conducted as, because of the numbers involved in the study and the nature of physical activity behaviour - that is, "maintainers" and "actioners" will naturally have larger variances in physical activity than "precontemplators", "contemplators", and "preparers"-it would be difficult to demonstrate the assumptions of normality and homogeneity of variance required for an analysis of variance. The Kruskal-Wallis test again indicated significant interstage differences $\left(H^{4}=31.86, \mathrm{p}<0.05\right)$. Follow up analysis with the Fisher multiple comparison test disclosed that there was no significant difference between precontemplators (PR) and contemplators (C) and between actioners (A) and maintainers (M). However, both actioners and maintainers reported significantly more physical activity than precontemplators (A $v$ PR: $95 \%$ confidence interval $(\mathrm{CI})=-1045$ to $-84 ; \mathrm{M} v \mathrm{PR}: 95 \% \mathrm{CI}=$ -1232 to -333 ) and contemplators (A $v \mathrm{C}$ : $95 \% \mathrm{CI}=-1029$ to $-213 ; \mathrm{M}$ v $\mathrm{C}: 95 \% \mathrm{CI}=$ -1210 to -468 ). Preparers (P) did not differ significantly from precontemplators, contemplators, or actioners but did from maintainers $(95 \% \mathrm{CI}=-952$ to -171$)$. These results indicate that SPAQ has concurrent validity with the stage of change model, as they show the expected relation between physical activity and

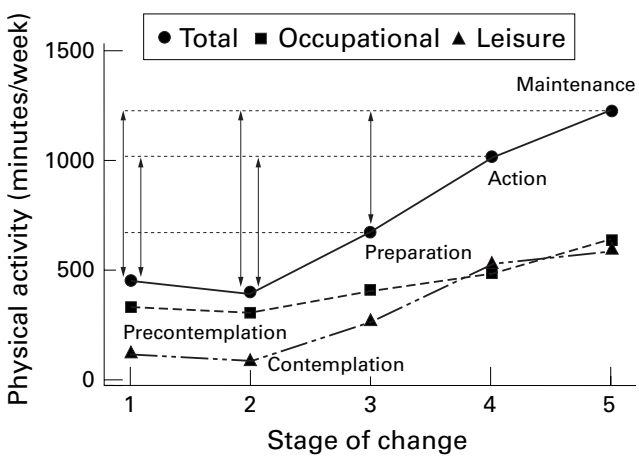

Figure 4 Relation between stage of exercise behaviour change and total, occupational, and leisure physical activity means (minutes a week). Double headed arrows indicate significant differences between stages for total physical activity $(p<0.05)$. stage of exercise behaviour change. Figure 4 shows this relation graphically.

Because of the differences between leisure time and occupational physical activity observed in study 1 , it was decided to analyse these two components separately. Figure 4 shows the relation between stage of exercise behaviour change and occupational and leisure time physical activity. Two one way analysis of variance tests disclosed no significant interstage differences for occupational physical activity but a significant difference for leisure time physical activity $(F[4,89]=13.14$, $\mathrm{p}<0.05)$. Follow up analysis with the Fisher multiple comparison test showed that leisure time physical activity had a similar relation to stage of exercise behaviour change as did total physical activity. The main conclusion that can be drawn from this and the lack of any significant interstage differences in occupational physical activity is that either the main differences in total physical activity between stages are primarily due to increases in leisure time physical activity or the questionnaire only has concurrent validity with the stage of change model for leisure time physical activity and not occupational physical activity. This point will be developed further in the discussion section.

Study 3

It was the aim of this study to validate SPAQ further against a more objective measurement of physical activity participation. A Caltrac motion sensor (Hemokinetics, Madison, Wisconsin, USA) was chosen for this purpose. These devices have been shown to provide a reliable and valid measure of physical activity in the laboratory, ${ }^{20}$ require relatively little time and effort on the part of the researcher or subject to operate, ${ }^{21}$ and are small and unobtrusive so they do not affect physical activity participation. ${ }^{21}$ However, there remains a question mark over their ability to function in a field study enviroment, ${ }^{22}$ but in the absence of an ideal measure of habitual physical activity, ${ }^{28}$ it was considered that the Caltrac would be the most suitable device for this study.

Thirty four people participated in the study. Eight (mean (SD) age 37 (11.17) years, all female) were regular members of a slimmers class, and twenty six (mean age 35 (13.5) years; 10 men, 16 women) were members of the public who had volunteered for a dietary analysis project.

Participants were asked to wear a Caltrac motion sensor for four consecutive days. The Caltrac motion sensor is able to estimate the total energy (kcal) used by the wearer over the period worn. After five days, participants were asked to complete a SPAQ. It was therefore possible to make a comparison between the Caltrac activity (kcal) and the amount of self reported physical activity (minutes) recorded for the four day period.

The relation between Caltrac activity and SPAQ was analysed using a test of association, the linear regression. The strength of the relation was determined using a correlation coefficient (it has to be noted that as the results obtained from Caltrac cannot be converted 
into minutes of activity, the limits of agreement technique $^{26}$ cannot be used to test the level of agreement between these two measurements).

From the original 34 participants, only 30 sets of data could be analysed because of one drop out, incomplete questionnaires, and a malfunctioning of the Caltrac. Initially, there was little or no correlation between the minutes of recorded physical activity and kilocalories on the Caltrac: $r=0.1294$. However, a review of the recorded data indicated that the walking at work component of the occupational physical activity section created data sets that were much higher than the rest, suggesting that it may have been overestimated. This concurs with study 1 , which showed that occupational walking was far less reliably recalled than the other sections of SPAQ. With this in mind, it was decided to analyse the relation between the Caltrac and SPAQ results without the occupational walking component; the correlation improved to $r=0.34$. In addition, on further analysis of the data, it appeared that four extreme sets of data existed. One of the participants (who was the heaviest at $203 \mathrm{lb}$ ) recorded a high activity kilocalorie count in comparison with recorded minutes. This may have had some bearing on the Caltrac's calculation of the resting metabolic rate, ${ }^{29}$ possibly underestimating it. On contacting the other volunteers for whom extreme sets of data were produced, it was found that one had injured her leg, and was carrying out resistant training for rehabilitation, which may not be detected by the Caltrac, ${ }^{28}$ and for the other two, errors were detected in the extreme values recorded. It was clear that major problems existed with the data for these four participants. Analysis of the relation between SPAQ (without occupational walking) and the remaining 26 data sets produced a correlation of $0.52(\mathrm{p}<0.05)$.

\section{Discussion}

Traditionally, the significant correlation coefficient (0.998) of test $v$ retest results for SPAQ as shown in study 1 would be cited as overwhelming evidence of the test-retest reliability of SPAQ. However, the coefficient of repeatability $(R)$, established using the technique of Bland and Altman, ${ }^{26}$ indicated a possible variation of up to 53 minutes in total physical activity using the same data. This study is therefore a clear example of the point being made by Bland and Altman. ${ }^{26}$ It is clear from fig 1 (and the significant correlation coefficient) that the two measurements are highly related, but it is also clear from fig 2 (and the coefficient of repeatability) that they are not in total agreement.

Study 1 found that leisure physical activity had an $R$ of 29.3 minutes but occupational physical activity had an $R$ of 54.6, this being mainly due to occupational walking which had an $R$ of 39.8 minutes which was the highest of any of the sections of SPAQ. However, this in itself does not make occupational walking "unreliable". Indeed, the test-retest correlation coefficient was 0.997 , which was highly significant. Clearly, this shows that even the most unreliable section of SPAQ shows a far stronger relation between repeated measures than other questionnaires that are considered reliablefor example, $r=0.84$ for Stanford physical activity recall ${ }^{24}$ reported by Williams et al. $^{22}$ This would suggest that SPAQ is in turn more reliable, but as we are not aware of similar questionnaires being analysed with the limits of agreement technique ${ }^{26}$ it is impossible to substantiate this conclusion. Indeed, it would be interesting to note what level of agreement existed between two measurements that elicited a correlation coefficient of 0.84 .

The methodology of this study allows us to be confident that any variations in physical activity from test to retest are caused by SPAQ, as we have analysed the same period of physical activity. In a comparable study, it was decided to readminster the questionnaire within one month of the original completion. ${ }^{22}$ Although we know that physical activity is relatively stable over this time period, how can we be sure that any differences observed were due to the questionnaire and not to differences in actual physical activity? A potential problem with the methodology used here is that only four days of a seven day recall questionnaire have been analysed. However, as the questions and recall procedures are identical for the three days not analysed with those for the four that were, it is reasonable to assume that a similar level of reliability would have been produced if we could have included these three days. Another potential problem is that completion of the retest SPAQ may have been affected by memory of completion of the first. Although we recognise that this could have occurred to some extent, this criticism could also be levelled at methodologies that require readministration within a few weeks. The methodolgy, used here, while potentially suffering the same "recall problem" as similar research, at least removes any potential problems caused by analysing two separate bouts of physical activity.

The first of the validity studies indicated that SPAQ has strong concurrent validity with the stage of exercise behaviour change model. Again it is interesting to note how each physical activity component (leisure and occupational) contributed to the interstage variations in total physical activity. It appeared that occupational physical activity did not significantly alter by stages of exercise behaviour change. However, actioners and maintainers reported significantly more leisure physical activity than precontemplators and contemplators. A possible explanation is that, because of the constraints of employment, most people are unable to accumulate significant amounts of physical activity while at work.

As already outlined, occupational walking was the least reliably recalled section of SPAQ. This question mark over the ability of SPAQ to produce an accurate record of this section was also evident in the final study, which attempted to validate the questionnaire against a more objective measure of physical activity. Only when this category was removed was a significant correlation found. It is evident that the occupational walking component of SPAQ needs to be revised. After communicating with participants in study 3, it appeared that the 
main problem with this category was that bouts of standing were being included. For example, a shop manager reported seven hours of walking at work. It is unlikely, knowing her environment, that she was walking for seven hours.

In the final study, there was initially a very low correlation coefficient of 0.13 when SPAQ was compared with a more objective measure of physical activity. There are several possible explanations for this. Firstly, as we have reported, question marks exist over the ability to record occupational physical activity with SPAQ. We were also aware that errors existed in the data of four of the respondents. Indeed, when corrections were made for both of these observations, a significant relation was found. In addition to the limitations of SPAQ (mainly occupational walking), Caltrac undoubtedly has its limitations for use in this kind of research. For example, some activities such as resistance training may not be detected, ${ }^{28}$ whereas they are are by the physical activity questionnaire. In addition, Tryon ${ }^{30}$ has shown that a number of subject characteristics may affect the Caltrac reading, and it has also been suggested that the precise placement of the unit is essential for accurate measurement. ${ }^{22}$ However, until an ideal physical activity measure for use in field studies is identified, the use of motion sensors is the next best thing.

\section{CONCLUSION}

SPAQ has been shown to be quick and easy to complete and practical for use with large subject numbers in a variety of situations (including postal surveys). It has been shown to be reliable and hold strong concurrent validity with the stage of exercise behaviour change model. The study has also shown that SPAQ holds criterion validity, but it has to be recognised that the evidence was not overwhelming, partly because of limitations in SPAQ and partly because of limitations in Caltrac. It is recommended that further work be conducted to refine SPAQ by improving its measurement of occupational walking. It is evident nonetheless that SPAQ can be used with confidence to measure outcomes in physical activity interventions when account is taken of its limitations.

The authors would like to thank both East Ayrshire Council and Forth Valley Health Board for their support throughout the course of this research.

1 Casperson CJ, Powell KE, Christenson GM. Physical activity, exercise and physical fitness: definitions and distinctions for health related research. Public Health Rep 1985;100:126-31

2 Berlin JA, Colditz GA. A meta-analysis of physical activity in the prevention of coronary heart disease. Am $\mathcal{F}$ Epidemiol 1990;132:612-28.

3 Fagard RH, Tipton CM. Physical activity, fitness, and hypertension. In: Bouchard C, Shephard RJ, Stephens T, eds. Physical activity, fitness, and health: international proceedings and consensus statement. Cha
4 Helmrich SP, Ragland DR, Leung RW, et al. Physical activity and reduced occurrence of non-insulin-dependent diabetes mellitus. N Engl F Med 1991;25:1-10.

5 Lee I-M. Physical activity, fitness, and cancer. In: Bouchard C, Shephard RJ, Stephens T, eds. Physical activity, fitness, and health: international proceedings and consensus statement. Champaign, IL: Human Kinetics Publishers, 1994:814-31.

6 Drinkwater BL. Physical activity, fitness, and osteoporosis. In: Bouchard C, Shephard RJ, Stephens T, eds. Physical activity, fitness, and health: international proceedings and consensus statement. Champaign, IL: Human Kinetics Publishers, 1994:724-36.

7 Rosenthal M, Haskell WL, Solomon R, et al. Demonstration of a relationship between level of physical training and insulin stimulated utilization in normal humans. Diabetes 1983;32:408-11.

8 Brownell KD. Exercise and obesity. Behav Med Update 1982;4:7-11.

9 Allied Dunbar, Health Education Authority, The Sports Council. The Allied Dunbar national fitness survey: a report on activity patterns and fitness levels. London: The Sports Council, 1992.

10 Northern Ireland Health and Activity Survey (1994). In: Health Education Board for Scotland. The promotion of physical activity in Scotland. A strategic statement, June 1995.

11 Health Education Board for Scotland, System 3 Scotland Panel Study (1993). In: Health Education Board for Scotland. The promotion of physical activity in Scotland. A strategic statement, June 1995 .

12 Prochaska JO, DiClemente CC. Stages and processes of self-change in smoking: towards an integrative model of change. F Consult Clin Psychol 1983;51:390-5.

13 Marcus BH, Rossi JS, Selby VC, et al. The stages and processes of exercise adoption and maintenance in a worksite sample. Health Psychol 1992;11:386-95.

14 Buxton K, Wyse J, Mercer T. How applicable is the stages of change model to exercise behaviour? A review. Health Education fournal 1996;55:239-57.

15 Pate RR, Pratt M, Blair SN, et al. Physical activity and public health: a recommendation from the Centres for Disease lic health: a recommendation from the Centres for Disease Sports Medicine. ҰAMA 1995;273:402-7.

16 Health Education Board for Scotland. The promotion of physical activity in Scotland. A Policy statement. Health Education for Scotland, 1997.

17 Loughlan C, Mutrie N. Recruitment of sedentary NHS staff for a workplace exercise programme using an adapted "stages of change" exercise questionnaire. I Sports Sci 1995;13:63-4.

18 Mutrie N, Loughlan C, Campbell M, et al. The transtheoretical model applied to four Scottish populations. F Sports Sci 1997;15:100.

19 Schoeller DA, Leitch CA, Brown C. Energy expenditure by doubly labelled water: validation in humans and proposed calculation. Am $\mathcal{F}$ Physiol 1986;250:R823-30.

20 Melanson Jr EL, Freedson PS. Validity of the Computer Science and Applications, Inc. (CSA) activity monitor. Med Sci Sports Exerc 1995;27:934-40.

21 Laporte RE, Montoye HJ, Casperson CJ. Assessment of physical activity in epidemiologic research: problems and prospects. Public Health Rep 1985;100:131-46.

22 Williams E, Klesges RC, Hanson CL, et al. A prospective study of the reliability and convergent validity of three physical activity measures in a field research trial. $f$ Clin Epidemiol 1989;42:1161-70.

23 Scottish Forum for Public Health Medicine. Scottish Needs Assessment Programme. Report: physical activity, University of Glasgow, 1996.

24 Blair SN, Haskell WL, Ho P, et al. Assessment of habitual physical activity by seven day recall in a community survey and controlled experiments. Am F Epidemiol 1985;122:79480

25 Loughlan CW, Mutrie N. An evaluation of the effectiveness of three interventions in promoting physical activity in a sedentary population. Health Education fournal 1997;56: 154-65

26 Bland JM, Altman DG. Statistical methods for assessing agreement between two methods of clinical measurement. Lancet 1986;1:307-10.

27 British Standards Institution. Precision of test methods. I: Guide for determination and reproducibility for a standard Guide for determination and reproducibility for a
test method. BS 5497, Part I. London: BSI, 1979.

28 Haskell WL, Yee MC, Evans A, et al. Simultaneous measurement of heart rate and body motion to quantitative physical activity. Med Sci Sports Exerc 1993;25:109-15.

29 Caltrac technical application note. Hemokinetics Inc., 5930 Seminole Centre Court, Madison, WI 53711, USA, 1993.

30 Tryon WW. Measurement of human activity. In: Tryon WW, ed. Behavioural assessment in behavioural medicine. New York: Springer, 1985:200-56

\section{Take home message}

It is now widely recognised that physical activity has many benefits, especially to health. Its accurate measurement is crucial to both physical activity research and promotion. SPAQ was designed to aid seven day total physical activity recall, and this study shows that it holds good test-retest reliability, concurrent validity, and limited criterion validity. 